\title{
Практический подход к проектированию заказных SMT-корпусов для ИС миллиметрового диапазона длин волн
}

\author{
К. Гомес-Дуарте ${ }^{1}$, А. Лезинов ${ }^{2}$
}

УДК 621.3.049.7| ВАК 05.27.01

\begin{abstract}
Многолетние исследования и разработки в области систем связи показали преимущества перехода на более высокие рабочие частоты. К наиболее значимым успехам, связанным с этими исследованиями, относятся: достижение более компактных схемных решений, увеличение коэффициента усиления антенн при сохранении их габаритов и резкое повышение пропускной способности при передаче данных. Тем не менее при реализации высокочастотных схем в реальных условиях остается много нерешенных сложных задач. Среди нетривиальных проблем следует выделить технологию корпусирования высокочастотных компонентов. В статье рассматриваются ключевые аспекты проектирования заказных SMT-корпусов, позволяющие обеспечить высокие характеристики ИС с рабочей частотой до 50 ГГц.
\end{abstract}

Д ля корпусов РЧ-компонентов критически важно чтобы в них могло быть использовано несколько схемных технологий одновременно, обеспечивая баланс характеристик и стоимости для конкретного приложения. Традиционные технологии изготовления корпусов, проверенные и отлично работающие на частотах до Х-диапазона, оказались неспособны обеспечить требуемые характеристики для Ис миллиметрового диапазона длин из-за влияния паразитных элементов и других технологических ограничений. Эти обстоятельства побудили разработчиков внедрять новейшие технологии при производстве корпусов, инновационные методы проектирования и усовершенствованные средства САПР для разработки экономически эффективных, масштабируемых решений для рынка ВЧ-техники и ВЧ-применений. Чтобы достичь высоких характеристик на частотах выше 55 ГГц сегодня отказываются от таких технологических решений, как заливка корпуса компаундом и длинные проволочные соединения, которые ухудшают параметры устройства.

\section{ВВЕДЕНИЕ}

Ожидается, что глобальный трафик мобильных данных вырастет с 11,2 петабайт/мес. в 2017 году до 48,3 петабайт/мес. в 2021-м. Для увеличения объема передаваемых данных в 1000 раз и поддержки семи миллиардов пользователей и семи триллионов устройств по всему миру была предложена перспективная технология сетей

Компания Mini-Circuits, инженер-разработчик Рч-схем. Компания «ЮЕ-Интернейшнл», руководитель группы ВЧ/СВЧ, alexey.lezinov@yeint.ru.
5G, обещающая сохранить на текущем уровне энергоэффективность и обеспечить практически нулевое время простоя.

Появление 5G активизировало работы в области интегральных схем, предназначенных для работы в ВЧ-диапазонах. Возникла также необходимость в разработке экономичных по стоимости корпусов, которые не только защитят ИС, но и обеспечат хорошие электрические характеристики в широком диапазоне рабочих частот.

Современные корпуса типа QFN для поверхностного монтажа не подходят для устройств миллиметрового диапазона. На более низких частотах не принимают в расчет паразитные элементы на пути прохождения сигнала, например разрывы в местах переходов от печатной платы к корпусу или от проводного соединения к кристаллу Ис. Однако такие неоднородности становятся важными, когда физические размеры элементов сравнимы с долями длины волны.

Другой недостаток QFN-корпусов - применение для герметизации прессования многокомпонентными компаундами, заливающими весь объем над кристаллом, что не только увеличивает электрические потери на более высоких частотах, но и делает невозможным монтаж кристалла с использованием воздушных перемычек между разваривающими кристалл проволочными соединениями. Кроме того, QFN-корпуса несовместимы с технологией перевернутого кристалла (flip-chip), поскольку параметры этих корпусов стандартизированы.

Был предложен целый ряд решений, направленных на устранение этих проблем. Например, QFN-корпуса с воздушной полостью допускают размещение кристаллов 
с воздушными перемычками, но они не обеспечивают хорошее согласование импедансов на высоких частотах. Микрокоаксиальные структуры допускают работу на высокой частоте, но требуют применения специализированных процессов сборки

Нестандартные решения для корпусирования могут компенсировать паразитные эффекты и допускают создание в корпусе воздушной полости. Следует отметить, что полностью заказные решения целесообразны, когда они реализуются в рамках быстрой, малорискованной стратегии проектирования, а также высокоавтоматизированного процесса сборки.

Современные радиочастотные приложения предъявляют жесткие требования к компонентам, помимо электрических характеристик. Высокая плотность монтажа сборок, высокая рабочая мощность и высокие требования по надежности заставляют конструкторов корпусов монолитных интегральных схем (МИС) СВч-диапазона искать оптимальное решение с точки зрения электрических, тепловых и механических характеристик.

Часто требуется найти компромиссное решение, поскольку конструктивные особенности, улучшающие одни характеристики, могут ухудшать другие. Например, решение, направленное на улучшение электрическиххарактеристик в ущерб более высокого рассеивания тепла может оказаться малополезным из-за повышения температуры проводников или полупроводников. По этой причине разработчикам важно учитывать одновременное влияние того или иного проектного решения на различные характеристики устройства.

В статье представлены результаты разработки, цель которой - создание заказных корпусов для поверхностного монтажа, обеспечивающих высокие характеристики ИС с рабочей частотой до 50 гГц (с учетом влияния печатной платы). Патентная заявка на эти решения находится на стадии рассмотрения.

\section{КОНСТРУКЦИЯ КОРПУСА}

В данной работе были исследованы корпуса двух типов керамический (LTCC) и из органического материала. Их поперечные сечения вместе с печатной платой показаны на рис. 1 и 2. Последующее описание относится к обоим вариантам. Кристалл ИС прикреплен к карману внутри подложки с помощью проводящей эпоксидной смолы. Такое решение минимизирует длину золотых проволочных выводов, которые соединяют контактные рч-площадки ис и корпуса, образуя низкочастотную цепь. В этой цепи проволочное соединение представляет собой сосредоточенную последовательную индуктивность $\mathrm{L}_{\mathrm{WB}}$, а контактные площадки корпуса и ИС - емкости $C_{\text {рк }}$ и $C_{\text {IC }}$ соответственно (рис. 3).

Правильная настройка этой согласующей цепи имеет решающее значение для точного согласования импедансов и достижения хороших электрических характеристик в широкой полосе частот. За контактной рЧ-площадкой корпуса следуют микрополосковая линия с волновым сопротивлением 50 Ом и согласованный вертикальный переход (переходное отверстие) к нижней площадке. Нижняя площадка корпуса выполнена с волновым сопротивлением 50 Ом в конфигурации с заземленным копланарным волноводом (GCPW). Корпус напаян на печатную плату, на которой реализован GCPW с волновым сопротивлением 50 Ом. Пластиковая или керамическая крышка крепится к корпусу непроводящей полуотвержденной (B-staged) эпоксидной смолой.

\section{ВЫБОР МАТЕРИАЛОВ}

Материалы и технология играют большую роль в достижении требуемых характеристик корпуса. Выбор подходящих материалов зависит от требований к приложению, таких как герметичность, максимальная рабочая частота, размер и вес корпуса, межсоединения первого и второго уровня, ограничения по тепловым режимам и допустимые вносимые потери межсоединений.

Для достижения желаемых рч-характеристик, как в LTCC-корпусах, так и в корпусах с органической подложкой, при выборе материала подложки нужно учитывать диэлектрическую проницаемость и тангенс угла потерь. Кроме того, подложка определяет топологию корпуса и совместимость с другими материалами.

Как уже отмечалось, в данной работе исследовались два вида подложек: LTCC и органическая. LTCC-корпус состоит из керамической монолитной структуры с полостью, сформированной в верхнихтрассировочныхслоях подложки (см. рис. 1). Открытая верхняя поверхность кармана покрыта сплошной металлизацией, соединенной с нижней площадкой заземления через несколько переходных отверстий. Проволочные соединения выполнены из жесткого материала, что облегчает их монтаж.

В корпусе с органической подложкой карман создается путем удаления части подложки и вскрытия металлизации в нижней части подложки (см. рис. 2), что улучшает качество заземления и снижает тепловое сопротивление.

Для обоих видов корпусов выбираются такие материалы и финишное покрытие проводников, чтобы получить хорошие РЧ-характеристики и обеспечить совместимость со стандартными сборочными процессами. Как правило, для LTCC-корпусов используют серебряные проводники с покрытием из иммерсионного золота с подслоем никеля (ENIG). Покрытие защищает серебряные проводники от окисления и должно быть совместимо с процессами пайки и проволочного монтажа.

В корпусе с органической подложкой используются медные проводники с различными вариантами покрытий, их выбор имеет решающее значение для высокочастотныхприложений, так как шероховатость поверхности 


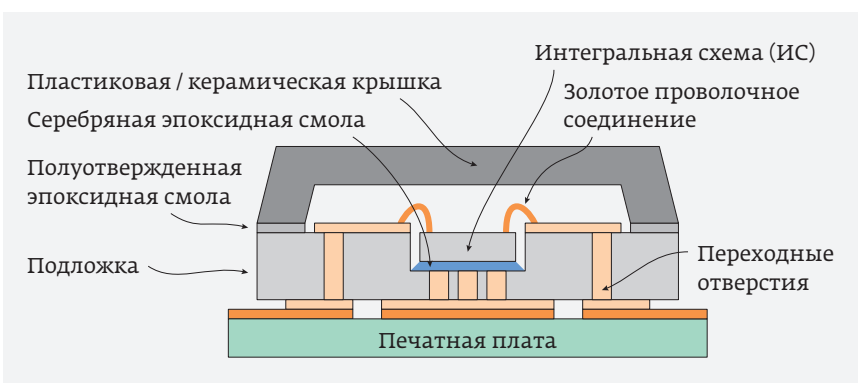

Рис. 1. Поперечное сечение керамического корпуса

и электрическая проводимость оказывают существенное влияние на вносимые потери.

Выбор проводящей эпоксидной смолы, используемой для монтажа кристалла МИС, влияет на общее тепловое сопротивление корпуса. Эпоксидная смола способствует рассеиванию большей части тепла, поскольку является основной точкой контакта кристалла и корпуса.

\section{ПРОЦЕСС МОДЕЛИРОВАНИЯ}

На этапе разработки анализировались электрические, тепловые и механические характеристики LTCC- и органического корпусов с применением мультифизического моделирования. В процессе моделирования применялось несколько симуляторов, которые работали последовательно. При этом полученные на каждом этапе результаты использовались в качестве входной информации для следующего симулятора.

Процесс моделирования включал несколько этапов.

1. Электромагнитное 3D-моделирование методом конечных элементов на упрощенном варианте геометрии конструкции (рис. 4). Результаты моделирования - данные об S-параметрах и пространственное распределение рассеиваемой мощности.

2. Тепловое 3D-моделирование методом конечных элементов на модели электромагнитной симуляции, дополненной геометрией, соответствующей тепловым и механическим (не электрическим) характеристикам. Как показано на рис. 5, основное внимание было уделено точному моделированию критических областей геометрии, таких как полые

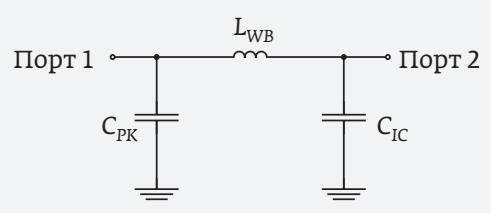

Рис. 3. Схематичное представление проволочного соединения в виде сосредоточенных элементов

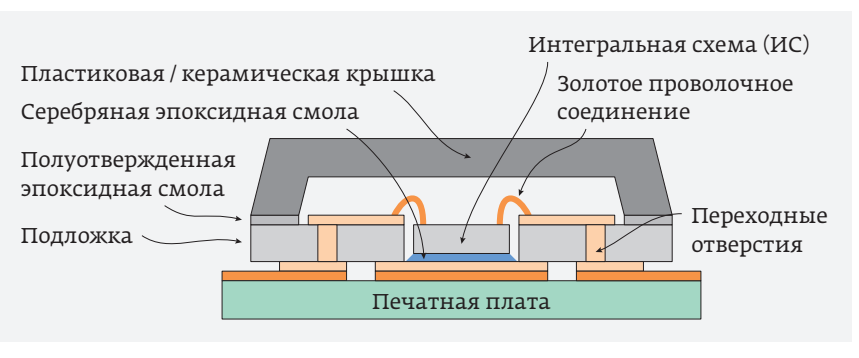

Рис. 2. Поперечное сечение корпуса из органического материала

и заполненные припоем металлизированные сквозные отверстия (РТН). Модель включает также припой и эпоксидную смолу для крепления кристалла. При моделировании использовались данные расчета рассеиваемой мощности, полученные при электромагнитном моделировании, в результате было получено распределение температуры в геометрии модели.

3. Механическое 3D-моделирование методом конечных элементов на полной геометрии модели с использованием пространственного распределения температуры в качестве части входных параметров симуляции. Результаты моделирования: механические напряжения и деформации в геометрии модели.

4. Вышеописанный процесс можно повторять до тех пор, пока не будут соблюдены критерии сходимости путем ввода на каждой итерации данных о повышении температуры и деформации геометрии модели в электрический симулятор. На практике для достижения полного совпадения результатов моделирования и физических измерений часто бывает достаточно одного прохода.

Будучи более сложным, чем процесс, включающий в себя отдельные задачи электрического, теплового и механического моделирования, мультифизическое моделирование дает инженерам целостное представление



Рис. 4. Электромагнитная модель LTCC-корпуса, которая включает только конструктивные элементы, имеющие отношение к электрическим характеристикам 


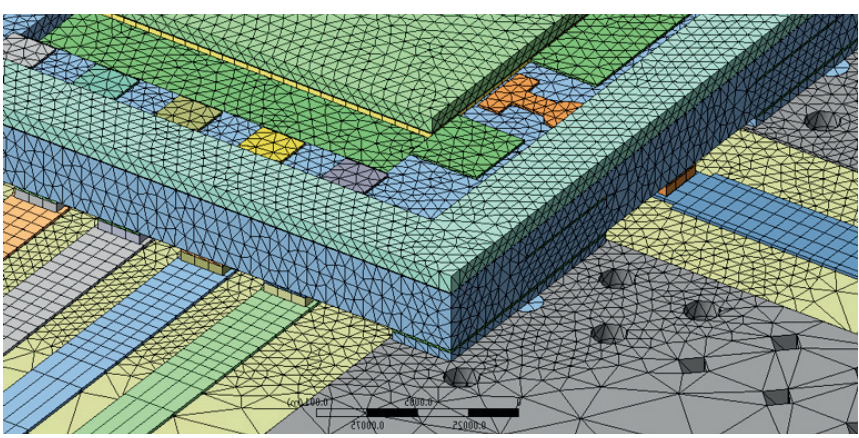

Рис. 5. Геометрия и сетка в увеличенном масштабе, используемые при тепловом и механическом моделировании LTCC-корпуса (крышка корпуса не показана)

о характеристиках разрабатываемого объекта. Например, при традиционном тепловом моделировании микрополоскового проводника обычно используется равномерно распределенный источник тепла в объеме или на поверхности проводника. Такой подход не учитывает ценную информацию о локальном выделении тепла из-за неоднородности плотности тока на частотах миллиметрового диапазона. Мультифизический подход к моделированию косвенно фиксирует этот и другие эффекты, не требуя специального внимания разработчика.

Способность мультифизического моделирования автоматически учитывать условия, которые слишком сложны для ручной настройки, особенно важна для LTCC-проектов. Tak как LTCC-объекты включают монолитную керамическую структуру со сложной геометрией внутренних проводников, тепловые изображения на внешней части такого устройства могут не полностью отражать его внутренние тепловые условия.

Поскольку электрические, тепловые и механические параметры проекта часто взаимосвязаны (из-за температурно-зависимых электрических сопротивлений, теплового расширения и т.д.), такой процесс моделирования позволяет лучше понять влияние проектных решений на совокупность характеристик. Предложенный процесс мультифизического моделирования, апробированный на многочисленных проектах с использованием различных технологий, позволил получить данные, очень близкие к реальным результатам измерений. Тем не менее этот процесс открыт для дальнейшего совершенствования.

\section{КАСТОМИЗАЦИЯ ИЛИ СТАНДАРТИЗАЦИЯ?}

Хотя корпус типа QFN - «рабочая лошадка» отрасли, используемая как для активных, так и для пассивных компонентов (вплоть до частот $\vee$-диапазона), он не оптимален для некоторых приложений из-за стандартизованных параметров. По мере освоения миллиметрового диапазона технологии корпусирования должны адаптироваться к быстро меняющимся потребностям отрасли.
В то время как универсальное решение может одинаково плохо подходить всем приложениям, полностью кастомизированное решение, дающее отличные результаты, может оказаться слишком дорогостоящим и потребовать значительных временных затрат. Для быстрой разработки экономически эффективного корпусного решения, в то же время обеспечивающего гибкость в применении, целесообразно объединить стандартные процессы и настраиваемые свойства проекта в рамках шаблона корпуса, адаптируемого под требования заказчика.

«Шаблонный» подход к проектированию корпуса позволяет многократно использовать проверенные элементы проекта, снижая затраты и риски в проектах, запускаемых с нуля. Этот подход предоставляет средства для адаптации к специфическим электрическим, тепловым, механическим и экологическим требованиям приложения, одновременно сводя к минимуму или устраняя необходимость в расширенной проверке новых проектных решений.

Корпуса типа QFN доступны в широкой номенклатуре стандартных размеров (3×3, 4×4 мм и т.д.), в то время как кристалл МИС может быть любых габаритов. Кристалл, слишком большой для стандартного QFN-корпуса, придется помещать в корпус большего размера, что потребует более длинных проволочных соединений с высокой паразитной индуктивностью. Сам корпус не компенсирует паразитные элементы, поэтому задача возлагается на геометрию проводников на печатной плате и кристалле.

Кроме того, в корпусах типа QFN для герметизации используется компаунд, который заполняет пространство вокруг выводной рамки, кристалла и проволочных соединений. Тонкие структуры на кристалле МИС, такие как воздушные перемычки, несовместимы с процессом заливки компаундом. Даже если хрупкие элементы МИС отсутствуют, герметик может нарушить или ухудшить характеристики чувствительной электроники просто из-за близкого расположения. Наконец, выводы QFN-корпусов в высшей степени стандартизированы, а возможность выбора размеров и геометрии контактных площадок ограничена. Для некоторых приложений паразитные элементы, связанные с фиксированной геометрией элементов корпуса, могут быть неприемлемыми.

Заказные LTCC-корпуса и корпуса с органической подложкой компании Mini-Circuits устраняют вышеуказанные ограничения, обеспечивая достаточно гибкие возможности для удовлетворения требований широкого спектра приложений. В этих корпусах кристалл расположен в кармане сверху подложки (см. рис. 1 и 2). Размеры кармана определяются габаритами кристалла заказчика, поэтому контактные площадки для проводных соединений могут быть размещены настолько близко к кристаллу, насколько это возможно, сводя к минимуму длину и индуктивность проводного соединения. 




a)

Рис. 6. 2-дБ аттенюатор в корпусе с органической подложкой: а - оценочная плата с установленным корпусом; 6 - корпус без крышки и flip-сhір-кристалл на подложке корпуса (крупным планом)

Таким образом, LTCC-корпуса и корпуса с органической подложкой обеспечивают большие возможности для выбора, несмотря на то, что в настоящее время они доступны в тех же размерах, что и стандартные корпуса QFN (3×3, 4×4 и 5×5 мм). Пластмассовая крышка крепится сверху кристалла и проволочных соединений с помощью полуотвержденной эпоксидной смолы, сохраняет над ними воздушный зазор, обеспечивая полугерметичное исполнение. Использование воздушного зазора вместо заливки герметизирующим материалом позволяет корпусировать хрупкие элементы МИС и минимизировать ухудшение электрических характеристик.

В отличие от корпусов QFN, LTCC-корпуса и корпуса с органической подложкой обеспечивают гибкость, которая требуется для наилучшего соответствия широкому спектру приложений. Включенные в конструкцию корпуса

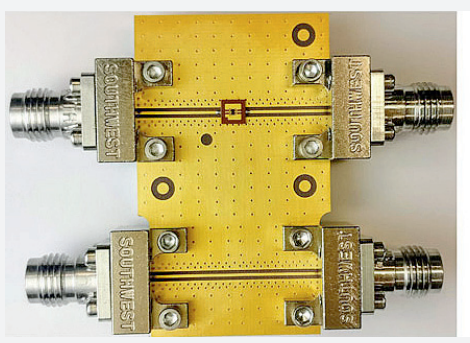

a)

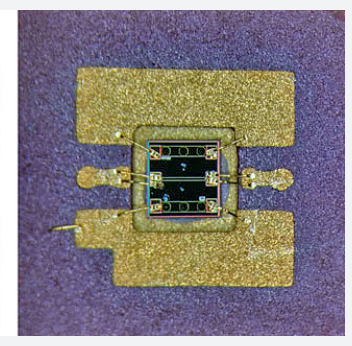

б)
Рис. 8. 2-дБ аттенюатор в керамическом корпусе: а - оценочная плата с установленным корпусом; б - корпус без крышки, flip-сhір-кристалл и проволочные соединения (крупным планом)

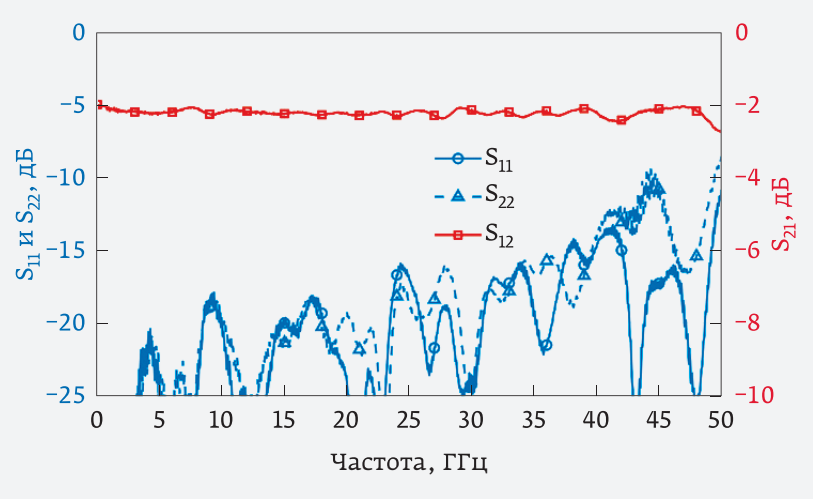

Рис. 7. Результаты измерений 2-дБ аттенюатора в корпусе с органической подложкой

перестраиваемые элементы электрически компенсируют паразитные элементы, связанные с переходными перемычками между печатной платой и корпусом и между корпусом и кристаллом МИС. Кроме того, поскольку корпус содержит печатные проводники, а не сплошную выводную рамку, посадочные места LTCC-корпусов и корпусов с органической подложкой можно адаптировать под нужды приложения с минимальными затратами на оснастку.

\section{ПРИМЕРЫ РЕАЛИЗАЦИИ}

Для проверки проектных решений и измерения характеристик было разработано, изготовлено и протестировано несколько корпусов с органической подложкой и LTCC-корпусов. Корпуса были смонтированы на оценочных печатных платах, выполненных на материале Taconic TLY-5 толщиной 0,127 мм с дорожками 50-Ом заземленного копланарного волновода (GCPW). Для сопряжения печатных плат с векторным анализатором цепей использовались 2,4-мм краевые разъемы от Southwest Microwave. Была выполнена стандартная



Рис. 9. Результаты измерений 2-дБ аттенюатора в керамическом корпусе 


\section{Mini-Circuits}

МИЛЛИМЕТРОВОГО ДИАПАЗОНА



\section{до 43,5 ГГц}


Усилители / Смесители/ Аттенюаторы

Неотражающие фильтры / Делители / Сумматоры Направленные ответвители / Умножители частоты

Доступны В пластиковых SMT корпусах и DIE форме

\section{С ЮЕ'ИНТЕРНЕЙШНЛ TPYIIA 10 :}

ЕДИНСТВЕННЫЙ ОФИЦИАЛЬНЫЙ ДИСТРИБЬЮТОР В РОССИИ
Санкт-Петербург (812) 313-34-40

Москва (495) 150-52-21
Екатеринбург (343) 365-90-40

Нижний Новгород (831) 220-59-64 


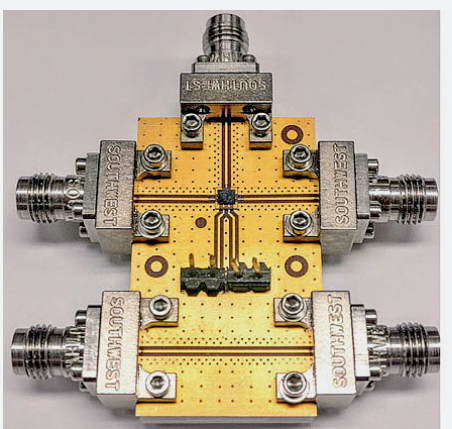

a)

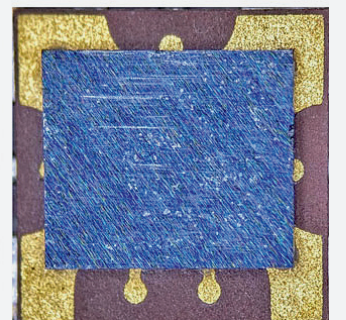

б)

Рис. 10. SPDT-ключ в керамическом корпусе: а - оценочная плата с установленным корпусом; б - корпус без крышки, flip-chip-кристалл и проволочные соединения (крупным планом)

SOLT-калибровка в диапазоне частот до 55 ГГц вплоть до базовой плоскости разъемов. Результаты измерения вносимых потерь для каждого корпуса были нормализованы путем вычитания потерь сквозной линии печатной платы.

\section{МИС 2-дБ аттенюатора в корпусе с органической подложкой}

В корпус с органической подложкой была установлена МИС 2-дБ аттенюатора и выполнен проволочный монтаж кристалла (рис. 6). На рис. 7 представлены результаты измерений. Коэффициент передачи $\mathrm{S}_{21}$ демонстрирует очень плоскую характеристику на уровне -2 дБ в диапазоне частот до 48 ГГц. Можно также отметить низкие обратные потери во всей полосе частот.

\section{МИС 2-дБ аттенюатора в керамическом корпусе}

мис 2-дБ аттенюатора в керамическом корпусе (рис. 8) также показала хорошие характеристики (рис. 9): плоскую характеристику $S_{21}$ на уровне -2 дБ в диапазоне частот до 55 гГц и низкие обратные потери во всей полосе частот.

\section{SPDT-ключ в керамическом корпусе}

В керамическом корпусе был смонтирован SPDT-ключ по технологии flip-chip (рис. 10). Результаты измерений устройства представлены на рис. 11, который иллюстрирует низкие обратные потери во всей полосе частот.

\section{ЗАКЛЮЧЕНИЕ}

Разработанные компанией Mini-Circuits корпуса двух типов, - использующие как LTCC, так и органические материалы, - продемонстрировали высокие электрические

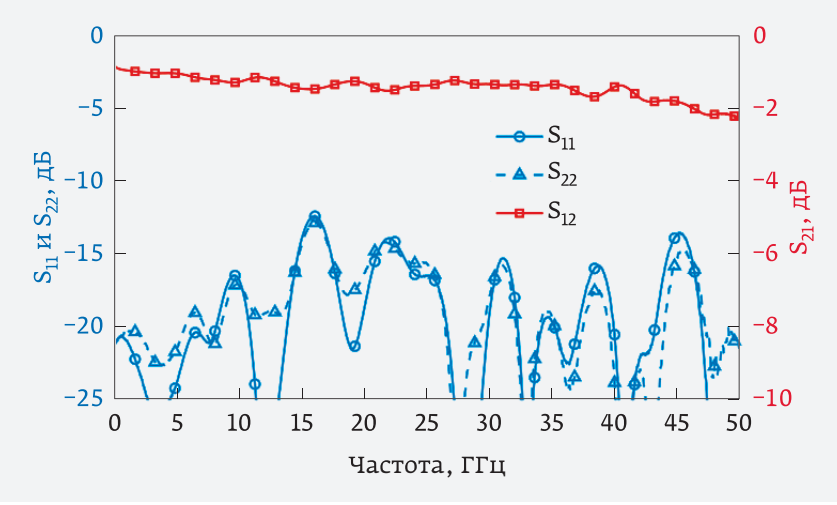

Рис. 11. Результаты измерений SPDT-ключа в керамическом корпусе

характеристики в полосе частот до 55 ГГц. Обе технологии корпусирования отвечают требованиям широкого спектра приложений, в частности, обеспечиваются хорошее согласование импедансов, переменные габариты кристаллов, широкий выбор числа контактных площадок ввода-вывода, типов сигналов (сигналы постоянного тока или РЧ-сигналы) и геометрии печатных плат. Подход компании Mini-Circuits к проектированию корпусов, сочетающий стандартные и настраиваемые свойства в корпусах-шаблонах, обеспечивает требуемые электрические характеристики и широкую применимость, минимизируя время, затраты и возможные риски.

\section{ЛИТЕРАТУРА}

1. $5 \mathrm{G}$ new radio: Introduction to the physical layer. National Instruments. (2018) // http://www.ni.com/enus/innovations/ wireless $/ 5 \mathrm{~g} / \mathrm{new}$-radio.html

2. Sanjuan E. A., Cahill S.S. QFN-based millimeter wave packaging to $80 \mathrm{GHz}$. - 2009 IEEE MTT-S International Microwave Workshop Series on Signal Integrity and HighSpeed Interconnects, pp. 9-1.

3. Fujii K., Morkner H. Two novel broadband MMIC amplifiers in SMT package for 1 to $40 \mathrm{GHz}$ low cost applications. - 2005 European Microwave Conference, vol. 2, pp. 4, pp. 1086.

4. Sturdivant R. Microwave and Millimeter-Wave Electronic Packaging. - Ser. Artech House microwave library. Artech House, 2013. - https://books.google.com.co/ books?id=xphQAgAAQBAJ

5. Coonrod J. Ambiguous influences affecting insertion loss of microwave printed circuit boards. - IEEE microwave magazine, vol. 13, no. 5, pp. 66-75, 2012.

6. Henry M., Free C., Reynolds Q., Malkmus S., Wood J. LTCC technology at high millimeter wave frequencies. - 2006 $1^{\text {st }}$ Electronic Systemintegration Technology Conference. IEEE, 200. - https://doi.org/10.1109/estc.200280087

7. Mini-Circuits. (2018) EP2KA+ Datasheet // https://www. minicircuits.com/pdfs/EP2KA+.pdf. 


\section{HAHEСЕНИE ГААЬВАНИЧЕСКИХ ПОКРЫТИЙ}

\section{из никеля (Ni) и золота (Au)}

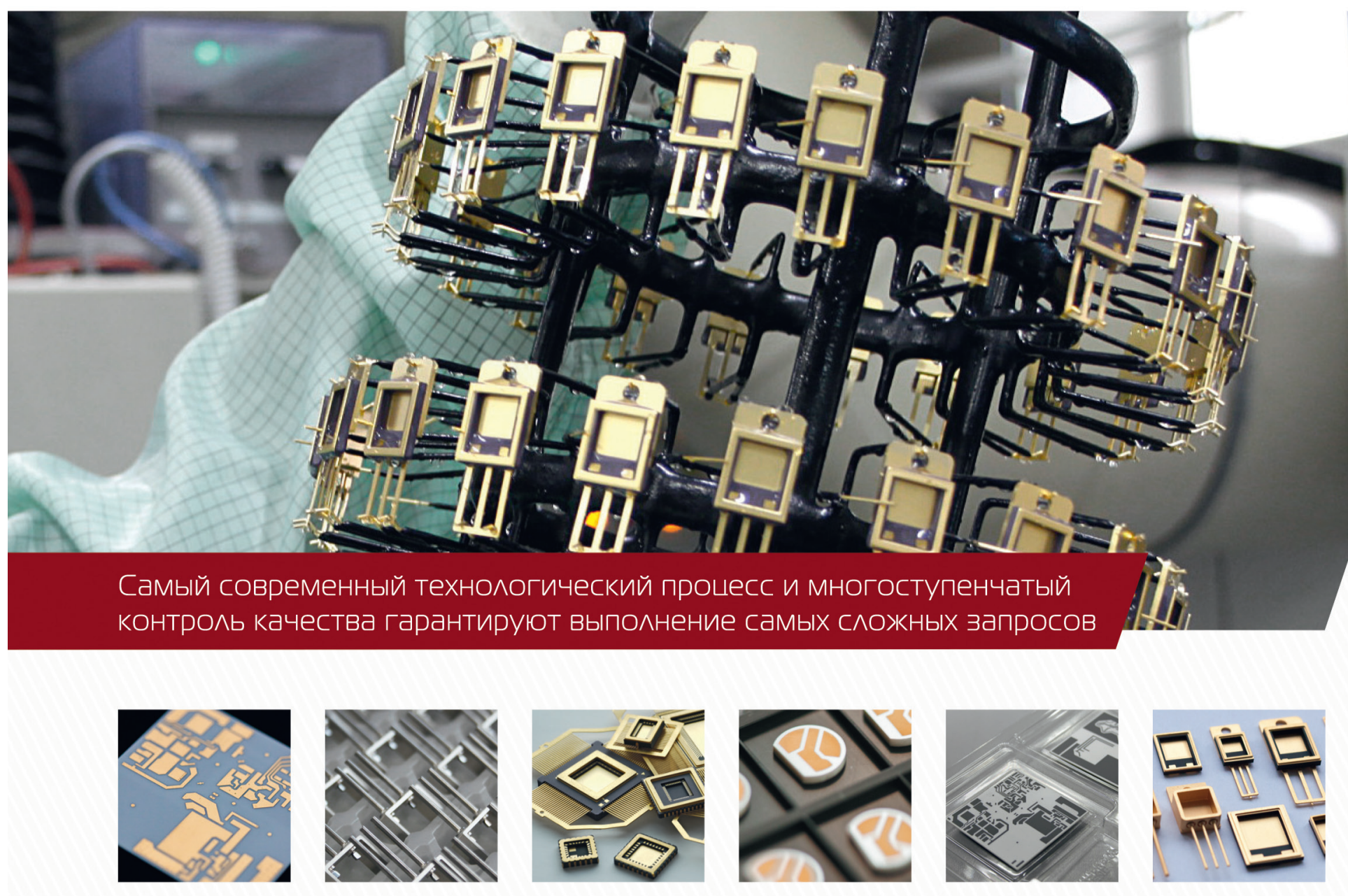

НА ПРОИЗВОДСТВЕ КОМПАНИИ «ТЕСТПРИБОР» ОСВОЕНЫ СЛЕДУЮЩИЕ ТЕХНОЛОГИИ НАНЕСЕНИЯ МЕТАЛЛИЧЕСКИХ ПОКРЫТИЙ:

- электрохимическое никелирование из электролитов различных составов

- электрохимическое никелирование сплавом никель-фосфор
- химическое никелирование сплавами никель-фосфор, никель-бор

- электрохимическое золочение

- химическое и иммерсионное золочение

- «черный никель» 